\title{
Basic biochemical and hematological parameters in perinatal asphyxia and their correlation with hypoxic ischemic encephalopathy
}

\author{
ANDREI IOAN MUNTEANU ${ }^{1}$, ANIKO-MARIA MANEA ${ }^{1}$, CRISTIAN MARIUS JINCA $^{2}$ and MARIOARA BOIA ${ }^{1}$ \\ Departments of ${ }^{1}$ Puericulture and Neonatology, ${ }^{2}$ Pediatrics, \\ 'Victor Babes' University of Medicine and Pharmacy, 300041 Timisoara, Romania
}

Received September 16, 2020; Accepted October 15, 2020

DOI: $10.3892 / \mathrm{etm} .2021 .9690$

\begin{abstract}
Perinatal hypoxic-ischemic encephalopathy (HIE) represents a major cause of neonatal death or long-term disability. Inflammation plays an important role in mediating brain damage induced by neonatal hypoxic-ischemic encephalopathy. The mechanisms underlying the inflammatory response in hypoxia and ischemia are complex and are still being extensively researched. The objective of this study was to determine the predictive value of peak lactate dehydrogenase (LDH), C-reactive protein (CRP), procalcitonin (PCT) and of the evolution of leukocytes, neutrophils and lymphocytes in the first $96 \mathrm{~h}$ after birth for the grade of encephalopathy and neurodevelopmental outcome in newborns with HIE. In order to reveal this relationship we used comparisons between the above mention parameters. The observed hematological changes were nonspecific. The vast majority of the 78 newborns included in the study had PCT values above normal in the first $24 \mathrm{~h}$, contrasting with CRP values that were positive in only $15.8 \%$ of the patients. A total of $76.9 \%$ of the patients had LDH values higher than the upper limit of normal values. The mean $\mathrm{LDH}$ values in patients with an unfavorable prognosis were 1,235 U/1. We can conclude that LDH is a good predictor of HIE in the first 12/24 h after birth.
\end{abstract}

Correspondence to: Dr Andrei Ioan Munteanu, Department of Puericulture and Neonatology, 'Victor Babes' University of Medicine and Pharmacy, Eftimie Murgu Square no. 2, 300041 Timisoara, Romania

E-mail: andrei.munteanu30@yahoo.com

Abbreviations: HIE, hypoxic-ischemic encephalopathy; LDH, lactate dehydrogenase; PCT, procalcitonin; CRP, C-reactive protein; CBC, complete blood count; Hb, hemoglobin; LE, leukocyte; NE, neutrophil; LY, lymphocyte, t1, time-point 1; t2, time-point 2

Key words: asphyxia, hypoxic-ischemic encephalopathy, newborn, premature, leukocyte, procalcitonin, lactate dehydrogenase

\section{Introduction}

Hypoxic-ischemic encephalopathy (HIE) is a major cause of neurologic disabilities in neonates despite the recent widespread use of hypothermia therapy. The incidence of HIE ranges from 1 to 8 per 1,000 live births in developed countries and is as high as 26 per 1,000 live births in underdeveloped countries (1). Hypoxia-ischemia (HI) is a contributing factor to neonatal morbidity and mortality, often leading to chronic neurological disorders and disabilities, such as mental retardation, motor and behavioral developmental issues, cerebral palsy, seizure, and epilepsy. Newborns with mild HIE (grade I) have a favorable evolution. Approximately $80 \%$ of the patients with grade II encephalopathy recover; however, the mortality rate is 3 and $20-45 \%$ have neurological sequelae. Patients with severe HIE (grade III) have a mortality rate of $50 \%$ and survivors present severe neurological consequences (2).

Manifestations of HIE involve heart rhythm disorders, basic acid balance disorders $(\mathrm{pH}<7.0$ or basic deficiency $\geq 12 \mathrm{mmol} / \mathrm{l}$ ), low Apgar index, amniotic fluid impregnated with meconium or the need for respiratory support in the first few minutes of postnatal life (3).

The redistribution of cerebral blood flow induced by asphyxia is the main post-asphyxiation change. Brain injury results from hypoxia and ischemia. As a result of asphyxia, cardiac output is compensated by redistribution, thus increasing cerebral blood flow. If hypoxia persists, this self-regulatory mechanism is no longer effective, resulting in decreased heart rate, with systemic hypotension and decreased cerebral flow leading to brain damage. At the cellular level, oxygen depletion blocks oxidative phosphorylation resulting in an anaerobic metabolism, which is energy inefficient, resulting in: i) Rapid depletion of phosphate reserves, including adenosine triphosphate, ii) accumulation of lactic acid and iii) inability to maintain cellular functions.

Oxidative stress, which is basically an alteration in the balance between antioxidants and prooxidants, needs to be considered, particularly in view of the existence of certain microcirculation deficits and endothelial dysfunction related to excess production of free radicals, which results in increased oxidative stress status (4).

In the first phase, an initial healing process begins in the first $60 \mathrm{~min}$ after the acute injury. In the latent phase (between 
the first and the sixth hour), oxidative metabolism inflammation and the continuation of apoptotic cascades take place. Six to $48 \mathrm{~h}$ after the hypoxic-ischemic incident, phosphate reserves are depleted, excitatory neurotransmitters and free radicals are released. In the third phase, a few months after acute ischemia, late cell death, remodeling of brain tissue and astrogliosis occur $(4,5)$. Multiorgan dysfunction represents a natural consequence of the redistribution of blood flow mechanism. Affected cells release intracellular enzymes, some of which are easy to measure, such as lactate dehydrogenase (LDH), which is present in most body tissues. Elevated levels of this enzyme have been reported after neonatal asphyxia. These aspects make this enzyme a potential predictor of the severity of hypoxic-ischemic injury in the postnatal period (6).

In clinical practice, biochemical evidence of multiorgan dysfunction is evident in over $70 \%$ of the cases of acute intrapartum asphyxia leading to neonatal encephalopathy (7). The measurement of LDH at $72 \mathrm{~h}$ may differentiate between neonatal asphyxia and other non-asphyxiating etiologies, especially in newborns with nonspecific signs of disease. In a study of 45 newborns, Reddy et al showed that LDH sensitivity was $100 \%$ and specificity was $89 \%$ (8). However, in another study of 61 newborns, it was shown that the sensitivity of LDH was $94 \%$ and the specificity was $67 \%$ (9).

In most organs, including the brain, neutrophils are the first cells to accumulate and infiltrate the tissue at reperfusion followed by monocytes and then T lymphocytes (10). Leukocyte recruitment into inflamed tissue proceeds in a cascade-like fashion. The first contact of neutrophils with the endothelium is mediated by selectins and their counterreceptors, followed by rolling of neutrophils along the endothelial wall of postcapillary venules and integrin-mediated arrest (11).

Cerebral ischemia induces an inflammatory response in both the parenchyma and the systemic circulation. Within hours after an insult to the brain, cytokines are produced in large amounts, and leukocytes are activated and migrate into the injured brain. There are few studies investigating the role of lymphocytes in HIE. A lymphocyte response is likely to be involved in the activation and exacerbation of chronic immuno-inflammatory after HIE. It is not clear yet whether this lymphocyte response improves, or, conversely, worsens healing after cerebral ischemia (12). In newborns with asphyxia at birth, a low number of lymphocytes have been found to be associated with a high risk of death (13).

Cytokines induce leukocyte proliferation, increase the number of circulating neutrophils and help endothelial transmigration and chemotaxis in damaged areas. It has been observed that the increased number of peripheral neutrophils in the first $96 \mathrm{~h}$ of life in newborns with HIE may contribute to the abnormal outcome of neurological development (14). However, in a study performed on 316 newborns, no associations were observed between changes in hematological values and brain damage due to post or intrapartum asphyxia (15).

This study was designed to investigate whether there is a relationship between serum cellular enzymes (LDH) with hematologic changes in red and white blood cells (lymphocytes and neutrophils) and the systemic inflammatory response revealed by procalcitonin (PCT) and C-reactive protein (CRP) values.
The first objective was to determine the evolution over time of hematological and biochemical changes in the newborn with HIE.

The second objective was to determine whether there was a relationship between the observed changes and the prognosis of newborns. The time to determine the blood tests varied between 6 and $96 \mathrm{~h}$. If blood tests were repeated, the maximum values in the first $96 \mathrm{~h}$ were recorded.

\section{Materials and methods}

Newborns. This is a retrospective, cross-sectional cohort study. This study was performed at the Neonatology Department at the Emergency Hospital for Children 'Louis Turcanu' Timisoara. This study was conducted over a period of 3 years, from January 1,2016 to December 31,2018. The study included 78 newborns weighing between $1 \mathrm{~kg}$ and $3.8 \mathrm{~kg}$ at birth.

The exclusion criteria from the study were: Incomplete patient data, information unavailable in the hospital's computer system, gestational age less than 35 weeks, newborns who suffered or had signs of infection/sepsis that could have influenced these investigations, the presence of a major congenital anomaly or any primary cause of encephalopathy other than ischemia/hypoxia.

Demographic, gestational, and perinatal data for the newborns included in the study were reviewed, including the presence of antenatal risk factors for both HIE, congenital anomalies and infections of any kind.

All newborns were identified by computer search of medical documents in the online database of the medical unit.

Hemotological investigation. Hematological investigations were performed with a Sysmex XS800i analyzer using impedance spectroscopy, flow cytometry, Hydro Dynamic Focusing (DC Detection method) and the reagents were provided by Sysmex Corp. (Kobe, Japan). The cell blood count (CBC) was collected from peripheral venous blood, $1 \mathrm{ml}$ of blood, and the sample was taken in a test tube with EDTA (sodium calcium edetate). The unit of measurement for $\mathrm{Hb}$ was $\mathrm{g} / \mathrm{dl}$ and for the leukocyte formula it was $\mu \mathrm{l}$ for biochemical investigations $1 \mathrm{ml}$ of peripheral venous blood was collected, using a normal test tube. The biochemical investigations were performed with a Cobas Integra 400 Plus analyzer and the reagents were provided by Roche Diagnostics GmbH. The methods used were turbidimetry for CRP and spectrophotometry for LDH. For PCT test, $1 \mathrm{ml}$ of peripheral venous blood was collected using an anticoagulant-free tube with separator gel. This investigation was performed on a Cobas e411 device and the reagents were provided by Roche Diagnostics $\mathrm{GmbH}$. The method used was ECLIA (immunochemistry with electrochemiluminescence detection). The units of measurement for CRP was $\mathrm{mg} / \mathrm{l}$, for PCT $\mathrm{ng} / \mathrm{ml}$ and for LDH U/l.

Analyzed biological markers were harvested at timepoint 1 (t1) which means blood tests were collected in the first hours after birth; and time-point 2 (t2) wich means blood tests were collected during the $96 \mathrm{~h}$ of follow-up.

Statistical analysis. Data analyses were performed using the statistical package (SPSS), version 23.0 (IBM, Corp.). Comparisons between group means were analyzed using the 
Table I. Baseline characteristics of the neonates $(\mathrm{N}=78)$.

\begin{tabular}{lc}
\hline Variables & Percentage \% \\
\hline Sex & 47.4 \\
Male & 52.6 \\
Female & 65.4 \\
Origin & 34.6 \\
Urban & 39.8 \\
Rural & 60.2 \\
Birth status & \\
Full-term & $2,311 \mathrm{~g}$ \\
Premature & 23.8 days \\
Average birth weight & 26.8 days \\
Average number of hospitalization days for the entire group & 9.7 days \\
Average number of hospitalization days for favorable prognosis group & \\
Average number of hospitalization days for unfavorable prognosis group & \\
\hline
\end{tabular}

ANOVA test. Pearson's Chi-squared test was used for each separate variable. If Pearson's Chi-squared test could not be used, the Fisher test was used. A P-value of $<0.05$ was considered to indicate a statistically significant difference.

\section{Results}

During the 3 years in which the study was performed at the Neonatology Department of the 'Louis Turcanu' Emergency Hospital for Children Timisoara, 2,191 newborns were admitted, of which 78 met the inclusion criteria. Of these, $52.6 \%(n=41)$ were female and $47.4 \%(n=37)$ were male. Regarding origin, $65.4 \%$ were from urban areas and $34.6 \%$ from rural areas. $60.2 \%$ were premature and $39.8 \%$ were full-term newborns. The average birth weight was 2,311 g. The average number of hospitalization days in the Neonatal Intensive Care Unit was 23.8 days. The average number of hospitalization days in the case of patients with a favorable prognosis was 26.8 days, and in the case of patients with an unfavorable prognosis was 9.7 days, these evolving to worse and then to death in a relatively short period of time (Table I).

The classification of patients was made according to prognosis. The prognosis was based on the following criteria: Birth weight, biological markers obtained, the patient's evolution during hospitalization and also the days of hospitalization in which they needed therapy. Depending on these 4 criteria, patients were considered to have a good or unfavorable prognosis. Patients with a negative prognosis had a lower average birth weight of 2,192 g compared to patients with a good prognosis, whose average was $2,338 \mathrm{~g}$.

Therefore, at discharge from the hospital, the patients status was as follows: $78.2 \%$ of patients were improved, $3.8 \%$ stationary, $1.3 \%$ aggravated and $16.7 \%$ were deceased. Depending on this status, we divided the patients into two groups: $82.1 \%$ with a favorable prognosis and $17.9 \%$ with an unfavorable prognosis.

Table II shows the average values of the results of the biological investigations gathered in the two groups under study and it can be seen that in the case of patients with unfavorable evolution, hematological parameters were lower and the average inflammatory sample was higher than that recorded in the patients with a favorable evolution.

Statistically significant differences were observed between the average hemoglobin $(\mathrm{Hb})$ values both at birth and in the first $96 \mathrm{~h}$ between the two groups studied; patients with an unfavorable prognosis having on average lower $\mathrm{Hb}$ values or a more pronounced decrease in the first days of life $(\mathrm{P}<0.01)$ (Fig. 1).

In the evolution of leukocytes (LE t2) $(\mathrm{P}<0.012)$, it was observed that in the case of patients with a unfavorable prognosis, LE values increased compared to birth values. The average variation of the values in the evolution of LE shows that in the case of patients with a favorable prognosis, LE values decreased by an average of 6,688 $\mu \mathrm{l}$ and in the case of patients with an unfavorable prognosis, LE values increased on average by 2,139 $\mu \mathrm{l}$ (Fig. 2). Regarding the evolution of NE values ( $\mathrm{t} 2)$, it was observed that patients with a favorable prognosis had a decreased evolution of NE compared to the value determined in the first hours of life (t1), unlike patients with an unfavorable prognosis, whose value had a slightly upward trend $(\mathrm{P}<0.002)$ (Fig. 3).

No statistically high values or variations were observed in LY values. In our study, the average LY values in the two groups of patients was approximately equal in the first $24 \mathrm{~h}$ and in evolution during the $96 \mathrm{~h}$ there was a slight decrease in values in patients with favorable evolution and a stagnation of values in the case of patients with unfavorable evolution (Table III).

In the evolution of inflammatory parameters of the CRP type $(\mathrm{t} 2)$, variations with significant values $(\mathrm{P}<0.001)$ were observed between the two groups studied; therefore, patients with a favorable prognosis had an average CRP values of $1.5 \mathrm{mg} / \mathrm{l}$ and those with an unfavorable prognosis-a value average of $19.82 \mathrm{mg} / \mathrm{l}$ (Fig. 4).

Statistically significant differences were not obtained in the case of PCT parameters. The average values (t1) in the case of patients with a favorable prognosis was $5.3 \mathrm{ng} / \mathrm{ml}$ and in the case of newborns with an unfavorable prognosis, it was $9.1 \mathrm{ng} / \mathrm{ml}$. In $\mathrm{t} 2$ the average values of patients with a favorable 
Table II. Prognosis according to the average values of biological markers at $\mathrm{t} 1$.

Favorable prognosis

\begin{tabular}{|c|c|c|c|c|c|c|c|}
\hline & $\mathrm{Hb}(\mathrm{g} / \mathrm{dl})$ & $\operatorname{LE}(\mu \mathrm{l})$ & $\mathrm{NE}(\mu 1)$ & $\mathrm{LY}(\mu 1)$ & $\mathrm{CRP}(\mathrm{mg} / \mathrm{l})$ & PCT (ng/ml) & $\mathrm{LDH}(\mathrm{U} / \mathrm{l})$ \\
\hline Mean & 17.09 & $18,955.00$ & $10,046.17$ & $4,565.10$ & 2.71 & 5.35 & 754.43 \\
\hline Std. error of mean & 0.299 & $1,447.89$ & 690.54 & 283.11 & 0.91 & 1.96 & 38.05 \\
\hline Std. deviation & 2.39 & $11,583.14$ & $4,734.12$ & $1,981.80$ & 7.23 & 13.34 & 304.40 \\
\hline
\end{tabular}

Unfavorable prognosis

\begin{tabular}{|c|c|c|c|c|c|c|c|}
\hline & $\mathrm{Hb}(\mathrm{g} / \mathrm{dl})$ & $\mathrm{LE}(\mu \mathrm{l})$ & $\mathrm{NE}(\mu 1)$ & $\mathrm{LY}(\mu 1)$ & $\mathrm{CRP}(\mathrm{mg} / \mathrm{l})$ & PCT (ng/ml) & $\mathrm{LDH}(\mathrm{U} / \mathrm{l})$ \\
\hline Mean & 14.90 & $15,947.85$ & $10,823.64$ & $4,208.33$ & 8.10 & 9.1 & $1,235.42$ \\
\hline Std. error of mean & 0.8224 & $1,719.48$ & $1,382.61$ & 508.46 & 4.62 & 3.8 & 255.32 \\
\hline Std. deviation & 3.0771 & $6,433.71$ & $4,585.60$ & $1,761.37$ & 17.29 & 13.8 & 955.33 \\
\hline
\end{tabular}

Hb, hemoglobin; LE, leukocytes; NE, neutrophils; LY, lymphocytes; CRP, C-reactive protein; PCT, procalcitonin; LDH, lactate dehydrogenase.

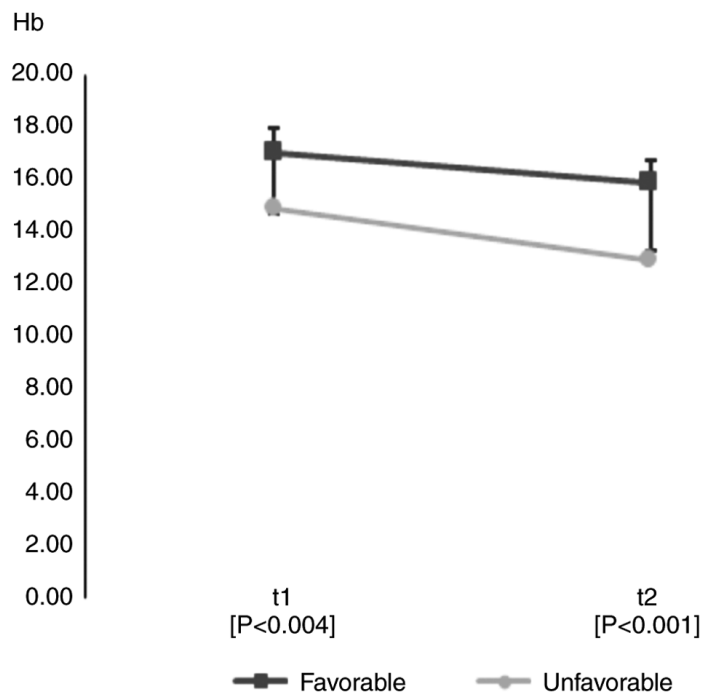

Figure 1. Evolution of hemoglobin ( $\mathrm{Hb})$.t1, time-point 1, blood tests conducted in the first hours after birth; $\mathrm{t} 2$, time-point 2, blood tests conducted during the $96 \mathrm{~h}$ of follow-up.

prognosis was $6.8 \mathrm{ng} / \mathrm{ml}$ and in the case of newborns with an unfavorable prognosis, it was $10.7 \mathrm{ng} / \mathrm{ml}$ (Table III). However, it was observed that $72.8 \%$ of patients had PCT values above normal. Making an analysis by risk groups, we observed that $71.7 \%$ of newborns with favorable prognosis had values of PCT above normal and $84.6 \%$ of newborns with unfavorable prognosis had PCT values above normal values.

In the newborns included in this study, it was observed that $76.9 \%$ of them had LDH values higher than the upper limit of normal values. With statistical significance $(\mathrm{P}<0.01)$, we can say that in the group with favorable prognosis, $73.4 \%$ of the newborns with HIE had LDH values above the maximum limit of normal, their average being around $754.43 \mathrm{U} / 1$. In the group with an unfavorable prognosis, $92.8 \%$ of the newborns with HIE had high values, the average value being 1,235.42 U/1 (Table III).

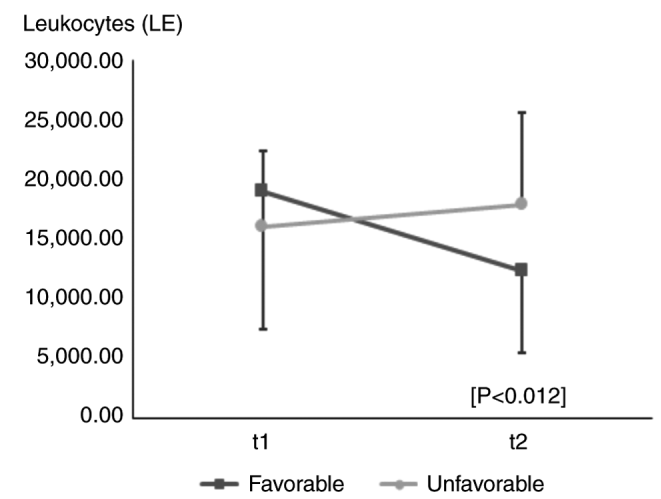

Figure 2. Evolution of leukocytes (LE).t1, time-point 1, blood tests conducted in the first hours after birth; $\mathrm{t} 2$, time-point 2, blood tests conducted during the $96 \mathrm{~h}$ of follow-up.

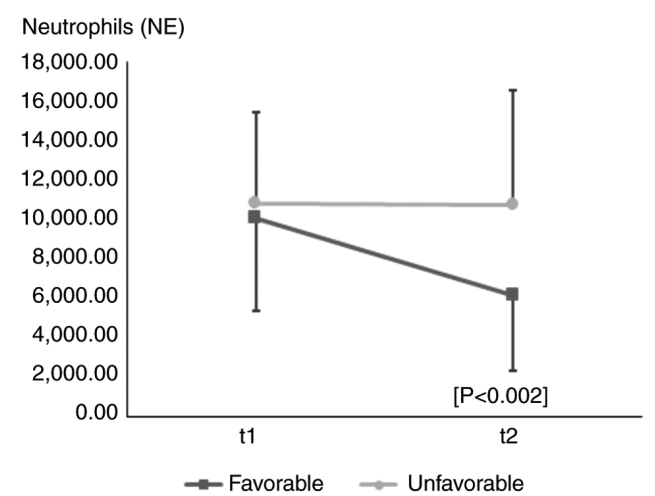

Figure 3. Evolution of neutrophils (NE).t1, time-point 1, blood tests conducted in the first hours after birth; $\mathrm{t} 2$, time-point 2, blood tests conducted during the $96 \mathrm{~h}$ of follow-up.

\section{Discussion}

Oxygen delivery to the brain is directly proportional to cerebral blood flow and arterial oxygen content and, therefore, 
Table III. Evolution of biological parameters.

\begin{tabular}{|c|c|c|c|c|c|c|c|}
\hline \multirow{2}{*}{$\frac{\text { Time }}{\text { t1 }}$} & \multirow{2}{*}{$\begin{array}{l}\text { Prognosis } \\
\text { Good }\end{array}$} & \multicolumn{2}{|c|}{ Mean $\mathrm{Hb}(\mathrm{g} / \mathrm{dl})$} & \multirow{2}{*}{$\frac{\text { Mean LY }(\mu 1)}{4,565.10}$} & \multirow{2}{*}{$\frac{\text { Mean PCT }(\mathrm{ng} / \mathrm{ml})}{5.35}$} & \multicolumn{2}{|c|}{ Mean LDH (U/l) } \\
\hline & & 17.09 & $\mathrm{P}<0.004$ & & & 754.43 & $\mathrm{P}<0.001$ \\
\hline & Unfavorable & 14.90 & & $4,208.33$ & 9.1 & $1,235.42$ & \\
\hline \multirow[t]{2}{*}{$\mathrm{t} 2$} & Good & 15.91 & $\mathrm{P}<0.001$ & $3,345.00$ & 6.8 & & \\
\hline & Unfavorable & 12.93 & & $4,358.00$ & 10.7 & & \\
\hline
\end{tabular}

Hb, hemoglobin; LY, lymphocytes; PCT, procalcitonin; LDH, lactate dehydrogenase.

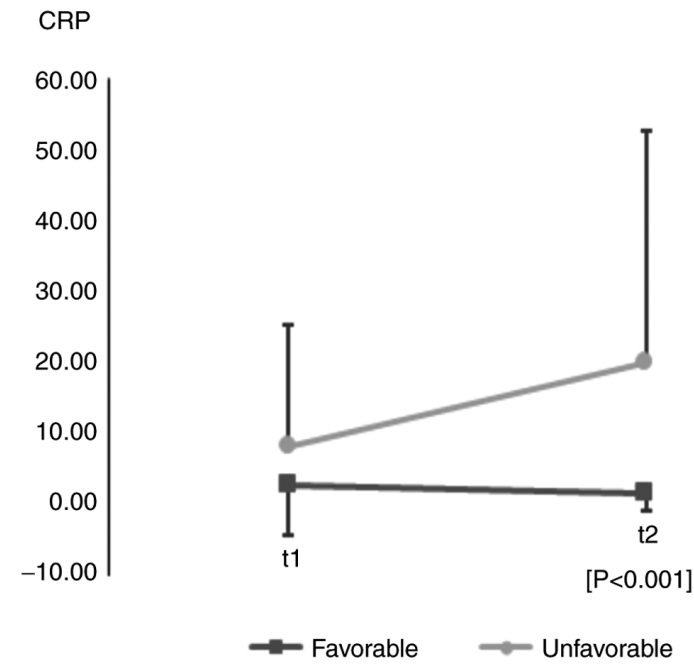

Figure 4. Evolution of the C-reactive protein (CRP) parameters. t1, timepoint 1 , blood tests conducted in the first hours after birth; 2 , time-point 2 , blood tests conducted during the $96 \mathrm{~h}$ of follow-up.

also to hemoglobin $(\mathrm{Hb})$ levels. According to this equation, a significant reduction in $\mathrm{Hb}$ may lead to decreased brain oxygen delivery and eventually tissue hypoxia, if the compensatory mechanisms aiming to keep a constant tissue oxygenation fail or are overtaken (15). One of the causes of moderate to severe perinatal asphyxia is perinatal anemia. Earlier research demonstrated that a lower level of initial $\mathrm{Hb}$ was a significant prognostic factor for abnormal neurodevelopmental outcome in severely anemic asphyxiated infants (16).

Cerebral hypoxia-ischemia enhances rapid expression of brain inflammatory cytokines and leads to an inflammatory cell response to injury that includes neutrophils, lymphocytes, and microglia (17). HIE was found not to be correlated with an increase in inflammatory samples, such as CRP, in all cases (18), which corresponds to the findings of our study, where only $15.8 \%$ of patients had elevated CRP values.

The observed hematological changes are attributable to asphyxiation injury, not brain damage, which was also noted in another study (19). It is possible that increased nucleated red blood cell production in the immediate neonatal state primarily reflects hypoxic injury (20).

Lymphocyte values in newborns have been shown to be dependent on the duration of fetal bradycardia. Fetal bradycardia exceeding $25 \mathrm{~min}$ resulted in an increase in the number of lymphocytes, the values being relatively transient with rapid normalization occurring between 18 and $24 \mathrm{~h}$ limiting its use as a marker for asphyxia. This variation in lymphocyte count has been attributed to a stress response that may originate in the thymus (21).

One study conducted in term infants with HIE found no correlation between lymphocyte number and outcome. Findings from another study revealed that elevated peripheral neutrophils were associated with worsened neurological outcome (22).

According to previous research as well as the present study it was also observed that lactate dehydrogenase (LDH) is a good predictor of HIE in the first 12/24 h after birth (23). In a retrospective study, serum LDH successfully predicted an abnormal mental or psychomotor development index at 18 months of age in neonates with HIE (24). This result is of clinical interest providing a potential prognostic marker, inexpensive and safe, in newborns with perinatal asphyxia. In addition, in a previously study, Karlsson et al obtained $100 \%$ sensitivity and $97 \%$ specificity in patients with HIE at a cut off level of $1049 \mathrm{U} / 1$ for LDH in the first $12 \mathrm{~h}$ after birth (6).

This retrospective study has several limitations. Firstly, infant blood tests were collected in the first hours after birth ( $\mathrm{t} 1$ ), then during the $96 \mathrm{~h}$ of follow-up ( $\mathrm{t} 2$ ), but were not harvested at the same time in all patients.

Larger groups of patients are needed in order to be able to say that the analysis of serum LDH has a high sensitivity and specificity for nerve cell and brain damage, because we know that LDH can be detectable in the cytoplasm of almost every cell in the human body and it becomes extracellular upon cell death. Considering its widespread presence in different types of tissues, increased LDH levels have been reported in many pathological conditions. LDH could be used as a biomarker of neuronal damage in the first hours of life, but perhaps for greater accuracy, it would be necessary to correlate with salivary LDH, which was not performed in our study.

The etiology of neonatal anemia could not be determined or well defined in some cases, here referring to maternal causes that could induce neonatal anemia because not all pregnancies were monitored by a gynecologist.

In conclusion, in patients with an unfavorable prognosis, the mean hemoglobin values were lower or had a more pronounced decrease in the first days of life. The values of leukocytes and neutrophils increased in the first $96 \mathrm{~h}$. A high sensitivity of PCT was observed in newborns with hypoxic distress and HIE. We can also conclude that LDH is a good predictor of HIE in the first 12/24 h after birth. 


\section{Acknowledgements}

Professional editing, linguistic and technical assistance was performed by Irina Radu.

\section{Funding}

No funding was received.

\section{Availability of data and materials}

The datasets used and/or analyzed during the current study are available from the corresponding author on reasonable request.

\section{Authors' contributions}

AIM and MB conceived and designed the study; AIM and AMM collected the data. AMM and CMJ analyzed the data; AIM and CMJ edited the figures and AIM, AMM and CMJ drafted the manuscript. $\mathrm{MB}$ revised the manuscript critically for important intellectual content. All authors contributed to the data interpretation and approved the submitted version.

\section{Ethics approval and consent to participate}

Approval of the local ethics committee (Ethics Committee for Scientific Research of the Emergency Hospital for Children 'Louis Turcanu'/approval no. 76/2020) was obtained prior to starting the study. Parental or caregiver consent was obtained where applicable. This publication and the database does not contain personal data, does not compromise anonymity or confidentiality or breach local data protection laws.

\section{Patient consent for publication}

Not applicable.

\section{Competing interests}

The authors declare that they have no competing interests.

\section{References}

1. Douglas-Escobar M and Weiss MD: Hypoxic-Ischemic Encephalopathy A review for the Clinician. JAMA Pediatr 169 397-403, 2015

2. Chaparro-Huerta V, Flores-Soto ME, Merin Sigala ME, Barrera de León JC, Lemus-Varela M de L, Torres-Mendoza BM de G and Beas-Zárate C: Proinflammatory cytokines, enolase and S-100 as early biochemical indicators of hypoxic-ischemic encephalopathy following perinatal asphyxia in newborns. Pediatr Neonatol 58: 70-76, 2017.

3. Allen KA and Brandon DH: Hypoxic ischemic encephalopathy: Pathophysiology and experimental treatments. Newborn Infant Nurs Rev 11: 125-133, 2011.

4. Serban D, Anton E, Chirita R, Bild V, Ciobica A, Alexinschi O, Arcan O, Popescu R, Paduraru L and Timofte D: Current aspects of the interactions between dementia, the brain renin-angiotensin system and oxidative stress. Arch Biol Sci 67: 903-907, 2015.
5. Yıldız EP, Ekici B and Tatlı B: Neonatal hypoxic ischemic encephalopathy: An update on disease pathogenesis and treatment. Expert Rev Neurother 17: 449-459, 2017.

6. Karlsson M, Wiberg-Itzel E, Chakkarapani E, Blennow M, Winbladh B and Thoresen M: Lactate dehydrogenase predicts hypoxic ischaemic encephalopathy in newborn infants: A preliminary study. Acta Paediatr 99: 1139-1144, 2010.

7. Hayes BC, Doherty E, Grehan A, Madigan C, McGarvey C, Mulavany S, Geary M, Matthews TG and King MD: Are serum markers of liver and muscle injury useful in neonatal hypoxic-ischemic encephalopathy? J Neonatal Perinatal Med 5: 305-310, 2012.

8. Reddy S, Dutta S and Narang A: Evaluation of lactate dehydrogenase, creatine kinase and hepatic enzymes for the retrospective diagnosis of perinatal asphyxia among sick neonates. Indian Pediatr 45: 144-147, 2008.

9. Jahan R, Khanam A, Masood A and Rehman R: Diagnostic accuracy of lactate dehydrogenase for diagnosis of perinatal asphyxia in neonates with non-reactive CTG. Pak J Med Heal Sci 13: 458-460, 2019.

10. Yilmaz G, Arumugam TV, Stokes KY and Granger DN: Role of T lymphocytes and interferon-gamma in ischemic stroke. Circulation 113: 2105-2112, 2006.

11. Zarbock A and Ley K: Mechanisms and consequences of neutrophil interaction with the endothelium. Am J Pathol 172: 1-7, 2008.

12. Liu F and Mccullough LD: Inflammatory responses in hypoxic ischemic encephalopathy. Acta Pharmacol Sin 34: 1121-1130, 2013.

13. Christensen RD, Baer VL, Gordon PV, Henry E, Whitaker C, Andres RL and Bennett ST: Reference ranges for lymphocyte counts of neonates: Associations between abnormal counts and outcomes. Pediatrics 129: e1165-e1172, 2012.

14. Morkos AA, Hopper AO, Deming DD, Yellon SM, Wycliffe N, Ashwal S, Sowers LC, Peverini RL and Angeles DM: Elevated total peripheral leukocyte count may identify risk for neurological disability in asphyxiated term neonates. J Perinatol 27: 365-370, 2007.

15. Lelubre C, Bouzat P, Crippa IA and Taccone FS: Anemia management after acute brain injury. Crit Care 20: 152, 2016.

16. Kalteren WS, Ter Horst HJ, den Heijer AE, de Vetten L, Kooi EMW and Bos AF: Perinatal anemia is associated with neonatal and neurodevelopmental outcomes in infants with moderate to severe perinatal asphyxia. Neonatology 114: 315-322, 2018.

17. Alsulaimani AA, Abuelsaad AS and Mohamed NM: Inflammatory cytokines in neonatal hypoxic ischemic encephalopathy and their correlation with brain marker S100 protein: A case control study in Saudi Arabia. J Clin Cell Immunol 6: 1-8, 2015.

18. Saito J, Shibasaki J, Shimokaze T, Kishigami M, Ohyama M, Hoshino R, Toyoshima K and Itani Y: Temporal relationship between serum levels of interleukin- 6 and C-reactive protein in therapeutic hypothermia for neonatal hypoxic-ischemic encephalopathy. Am J Perinatol 33: 1401-1406, 2016.

19. Shah V, Beyene J, Shah P and Perlman M: Association between hematologic findings and brain injury due to neonatal hypoxic-ischemic encephalopathy. Am J Perinatol 26: 295-302, 2009.

20. Vandana V, Amit V, Meena V, Anuradha B, Vivek B, Deepak V and Salone MR: Study of basic biochemical and haematological parameters in perinatal asphyxia and its correlation with hypoxic ischemic encephalopathy (HIE) Staging. J Adv Res Biol Sci 3: 79-85, 2011.

21. Dina P and Muraskas JK: Hematologic changes in newborns with neonatal encephalopathy. Neoreviews 19: e29-e33, 2018

22. Povroznik JM, Engler-Chiurazzi EB, Nanavati T and Pergami P: Absolute lymphocyte and neutrophil counts in neonatal ischemic brain injury. SAGE Open Med 6: 2050312117752613, 2018.

23. Yum SK, Moon CJ, Youn YA and Sung IK: Changes in lactate dehydrogenase are associated with central gray matter lesions in newborns with hypoxic-ischemic encephalopathy. J Matern Neonatal Med 30: 1177-1181, 2017.

24. Mehta A, Chawla D, Kaur J, Mahajan V and Guglani V: Salivary lactate dehydrogenase levels can provide early diagnosis of hypoxic-ischaemic encephalopathy in neonates with birth asphyxia. Acta Paediatr 104: e236-e240, 2015. 